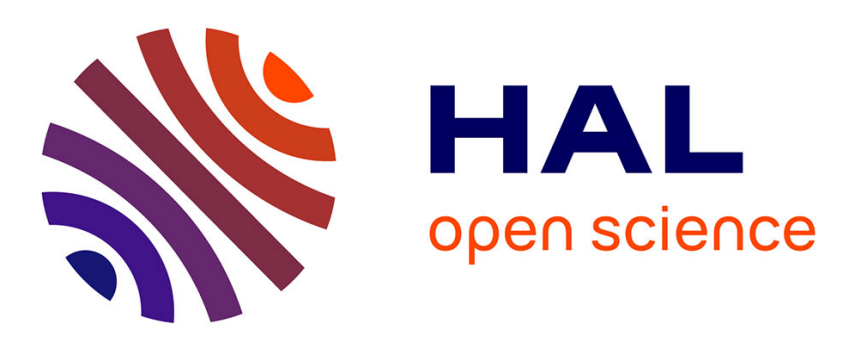

\title{
La netnographie : outil de prospective des métiers, une application aux nouveaux métiers du Web
}

\author{
Maria Mercanti-Guérin
}

\section{To cite this version:}

Maria Mercanti-Guérin. La netnographie : outil de prospective des métiers, une application aux nouveaux métiers du Web. Revue management \& avenir, 2009, 25 (5), pp.386. 10.3917/mav.025.0386 . hal-02055031

\section{HAL Id: hal-02055031 \\ https://hal.science/hal-02055031}

Submitted on 2 Mar 2019

HAL is a multi-disciplinary open access archive for the deposit and dissemination of scientific research documents, whether they are published or not. The documents may come from teaching and research institutions in France or abroad, or from public or private research centers.
L'archive ouverte pluridisciplinaire HAL, est destinée au dépôt et à la diffusion de documents scientifiques de niveau recherche, publiés ou non, émanant des établissements d'enseignement et de recherche français ou étrangers, des laboratoires publics ou privés. 
La prospective des métiers au service du management et de la GRH

\title{
LA NETNOGRAPHIE : OUTIL DE PROSPECTIVE DES METIERS, UNE APPLICATION AUX NOUVEAUX METIERS DU WEB
}

\author{
Maria Mercanti-Guérin \\ Maître de conférences \\ Université d'Evry Val d'Essonne \\ 2 rue du Facteur Cheval 91000 Evry (France) \\ Centre de recherche DMSP, DRM (CNRS UMR 7088), Université Paris Dauphine \\ 0146640662 maria.guerin@laposte.net
}




\title{
LA NETNOGRAPHIE : OUTIL DE PROSPECTIVE DES METIERS, UNE APPLICATION AUX NOUVEAUX METIERS DU WEB
}

\section{Résumé}

L'objectif de cet article est de décrire et comprendre les implications que peuvent avoir les blogs de salariés dans une démarche de prospective des métiers. L'utilisation de la netnographie nous a permis d'établir une taxinomie des nouveaux métiers du Web. L'article montre l'intérêt de coupler la méthode des scénarios comme outil de prospective et la netnographie. Cette recherche décrit pas à pas la démarche de recherche adoptée: l'application de la netnographie à une vision prospective.

Mots Clés : Blog, Prospective des métiers, Netnographie

\begin{abstract}
The purpose of this communication is to describe and understand the implications of workers blogs on jobs prospective. We develop netnography as an online research technique for providing taxonomy of new jobs of the Web. This article aims at showing the interest of using scenarios as prospective tool in a context of netnography. The article offers step-by-step instructions for completing a netnography research project in a prospective vision.
\end{abstract}

Key words : Blog, Jobs prospective, Netnography 


\section{Introduction}

Manifestation apparue avec le Web 2.0, les blogs de salariés se multiplient sur la toile. Faisant partie des réseaux sociaux, les blogs constituent un observatoire de premier ordre des pratiques professionnelles. Néanmoins, très peu de recherches portent sur ce phénomène. Sa récence et la difficulté de développer des méthodes d'études adaptées expliquent la faible production scientifique qui leur est consacrée. Pourtant, une méthode initialement qualitative et utilisée en marketing, la netnographie ou ethnographie appliquée à Internet représente une piste de recherche intéressante.

Pouvant être assimilés à des communautés de pratique, les blogs de salariés présentent bon nombre de caractéristiques similaires à ce concept. Une communauté de pratique est définie « comme un réseau d'individus socialement liés, engagés dans une activité, une pratique conjointe ou complémentaire, partageant un langage, des préoccupations, une passion, des concepts et qui développent leurs compétences par l'échange et des activités communes de résolution de problèmes »(Grimand, 2006). Liées aux réflexions portant sur la gestion de la connaissance et l'apprentissage, les communautés de pratique montrent que la socialisation et l'interaction entre membres influent sur la production des savoirs (Wenger et Snyder, 2000). Par ailleurs, elles remettent au cœur du débat scientifique portant sur les liens communautéconnaissance le principe de l'informel comme espace productif privilégié. Les théoriciens des communautés de pratique parlent «d'espaces interstitiels » pour définir des lieux de dialogue implantés au cœur même des failles de l'organisation. A ce titre, les blogs de salariés sont identitaires et donc constructeur de sens tout en permettant de s'affranchir des «tentations normatives » (Grimand, 2006) liées à la récupération managériale dont font l'objet les communautés de pratique. Néanmoins, les blogs de salariés ne sont pas qu'identitaires. Ils sont enracinés dans la sphère professionnelle. A ce titre, ils représentent un témoignage multiforme du quotidien des salariés. En outre, leur typologie permet de mieux comprendre l'importance de la prospective en leur sein. La description qu'ils font de leur métier s'articule autour d'un axe temporel assez peu axé sur le descriptif du passé, très ancré dans le présent et se voulant résolument projeté vers l'avenir. Comme le souligne Montil (2001), les ressources humaines peinent à intégrer la prospective des métiers dans ses pratiques de gestion. Même la GPEC (gestion prévisionnelle des emplois et des compétences) s'inscrit dans une « conception restrictive de la compétence essentiellement statique » (Montil, 2001).

Parallèlement, certains salariés reprennent à leur compte par des démarches strictement individuelles la nécessité de penser les «futurs possibles » de leurs métiers. A cela, plusieurs explications possibles. La première tient à l'inquiétude générée par les mutations de leurs métiers, mutations auxquelles ils doivent s'adapter afin de préserver «leur employabilité ». La deuxième explication est liée à l'esprit « poste avancé » qui anime certains blogs. Tenus par des salariés, qui par leur démarche se positionnent comme des acteurs-experts ${ }^{1}$ tels qu'ils sont définis par Boyer et Scouarnec (2008), les blogs de salariés renforcent l'identification au métier, mettent en avant les compétences dans ce qu'elles ont d'individuel, diffusent les bonnes pratiques au sein des différents corps de métier et favorisent la transversalité en offrant des espaces ouverts aux salariés d'une même entreprise.

Notre communication s'attache donc dans un premier temps à proposer une typologie des blogs de salariés comme une étape indispensable à la compréhension du phénomène. Cette typologie s'est effectuée en utilisant la netnographie dont nous présenterons les principales

\footnotetext{
${ }^{1}$ « La prospective des métiers repose sur une méthodologie active reposant sur la participation des personnes qui exercent le ou les métiers analysés -les acteurs-experts-. Ces derniers, à la différence des démarches de gestion par les compétences, sont alors acteurs des changements à venir. » (Boyer et Scouarnec, 2008, p 106).
} 
caractéristiques. Dans un second temps, nous montrerons, à travers une étude de cas réalisée sur les nouveaux métiers du web, la capacité des blogs de salariés à appréhender les évolutions de leur environnement et à s'interroger sur ses mutations. L'application de la méthode des scénarios couplée à la netnographie permet de décrire un certain nombre de futurs possibles qui pourraient s'appliquer à ces métiers émergents. Nous conclurons par une réflexion sur les apports et les limites de cette nouvelle méthode de recherche.

\section{Les blogs de salariés, qui sont-ils ? Essai de typologie}

Afin de mieux comprendre le fonctionnement et les apports des blogs de salariés en matière de prospective, nous avons souhaité rappeler la typologie des blogs de salariés qui a servi de base à la structuration de notre terrain. Cette typologie est issue d'une étude exploratoire effectuée sur une quarantaine de blogs de salariés (Mercanti-Guérin, d'Andria, 2008).

Deux sources de la littérature ont servi de base à son élaboration. La première source de littérature est celle de Ross (2005). Ross propose une typologie des blogs pouvant avoir un impact sur les politiques RH fondée sur trois axes : l'axe organisationnel, l'axe professionnel et l'axe personnel. Le blog organisationnel repose sur une forte implication de l'entreprise dans la création et la gestion des blogs. Les raisons d'être du blog vont de la facilitation de la communication interne à la transmission des savoirs et des expertises au sein des salariés ou à la maîtrise de la communication corporate auprès des parties prenantes. Le blog professionnel est souvent organisé par l'entreprise tout en laissant une relative liberté aux contenus mis en ligne. Nous citerons le cas d'IBM, qui, au sein de son site, réserve un espace libre aux blogs de ses salariés-experts ${ }^{2}$. Le blog personnel a pour caractéristique d'être totalement indépendant de l'entreprise. De ce fait, les phénomènes d'hostilité à cette dernière sont plus fréquents.

La seconde est issue des réflexions des blogueurs sur ce phénomène. Plusieurs, dont Loic Le Meur (2005), un des blogueurs français les plus connus proposent une classification des différents types de blogs tenus par des salariés. L'étude de la taxinomie des blogs de salariés vue par la blogosphère nous permet de distinguer différentes typologies :

- Les blogs internes créés pour faciliter la gestion de projet et dont la caractéristique première est de permettre le partage des compétences

- Les blogs collaboratifs regroupant une profession et dont l'objectif est le partage des expériences ou la fédération des intérêts

- Les blogs introspectifs de salariés qui racontent leur quotidien en entreprise

- Les blogs « comité d'entreprise » qui en dehors du comité d'entreprise officiel rendent compte de la vie sociale de ses employés (voyages, naissances, pots de départ...)

- Les blogs des syndicats qui ouvrent un large espace de discussion aux salariés

- Les blogs de réaction créés par les salariés sans passer par les structures syndicales observés lorsque l'entreprise traverse une grave crise sociale

\subsection{Une application de la nethnographie dans un contexte $R H$}

Ces propositions de typologie ont servi de point de départ à une démarche plus structurée consistant à étudier les blogs de salariés sous l'angle de la netnographie. La netnographie peut se définir comme l'ethnographie appliquée à Internet. Cette méthode initialement qualitative adapte les techniques de recherche ethnographiques aux communications en ligne.

\footnotetext{
${ }^{2}$ L'entrée sur les blogs des salariés-experts d'IBM se fait à partir du site de ce dernier. Il présente pour chacun son nom, sa photo, son parcours et son expertise technique ainsi que les thématiques de ces billets. Avant de télécharger le blog choisi, IBM précise que l'internaute sort du site officiel d'IBM.
} 
Initialement utilisée en marketing pour la richesse d'observations qu'elle apporte (forums de discussion, blogs...) la netnographie est encore très rarement utilisée dans un contexte RH. Le recours à cette méthode devrait suivre dans le futur le développement croissant de l'e-RH. Son avantage est de conférer au chercheur une position d'observateur n'influant pas sur le participant, ce qui en fait une méthode non intrusive. Plus généralement, la netnographie s'attache à la connaissance du local, du particularisme et du spécifique (Kozinets, 2002). Füller et al. (2008) identifie 5 étapes structurant la méthodologie de la netnographie.

1. Détermination des objectifs de la recherche et du profil idéal des membres de la communauté

2. Identification et sélection des communautés

3. Observation et collecte des données

4. Analyse des données et interprétation des résultats

5. Transfert des résultats en solution

\subsection{Champ de recherche et méthodologie}

Les profils des blogs retenus sont détaillés dans le tableau 1. Notre terrain de recherche est composé de 36 blogs de salariés. Ce corpus (identification et sélection des communautés) a été constitué par :

- Une recherche grâce aux liens hyper-textes présents sur les billets des blogueurs analysant le phénomène et renvoyant aux blogs de salariés les plus fréquentés ou les plus représentatifs de leur catégorie

- Une recherche sur les wikis, moteurs de recherche spécialisés à travers des mots clés pointés dans les blogs

- Une syndication systématique via les flux RSS afin de suivre de façon longitudinale certains blogs

Une grille de classification des blogs (observation et collecte des données) a été réalisée à partir de la littérature et de l'aide d'un expert travaillant dans le domaine des portails. Les critères suivants ont été retenus comme critères de classification des différents blogs de l'échantillon :

Tableau 1 : critères de classification

\begin{tabular}{|l|l|l|l|l|l|l|}
\hline Blogueur(s) & $\begin{array}{l}\text { Salarié } \\
\text { isolé } \\
\text { identifié3 }\end{array}$ & $\begin{array}{l}\text { Salarié } \\
\text { isolé } \\
\text { anonyme }\end{array}$ & $\begin{array}{l}\text { Regroupement } \\
\text { de salariés } \\
\text { d'une même } \\
\text { profession }\end{array}$ & $\begin{array}{l}\text { Regroupement } \\
\text { de salariés d'un } \\
\text { même secteur } \\
\text { d'activité }\end{array}$ & $\begin{array}{l}\text { Billets de } \\
\text { salariés au } \\
\text { sein de } \\
\text { blogs tenus } \\
\text { par des } \\
\text { syndicats }\end{array}$ & $\begin{array}{l}\text { Regroupement } \\
\text { de salariés au } \\
\text { sein d'une } \\
\text { même entreprise }\end{array}$ \\
\hline
\end{tabular}

\footnotetext{
${ }^{3}$ Dans certains cas, le blogueur est clairement identifié par son nom. Dans d'autres cas, ni le nom du blog, ni son contenu ne permettent d'identifier le blogueur (salarié anonyme). L'anonymat introduit, comme nous le verrons dans la typologie, un rapport différent à l'entreprise avec une part plus importante des billets consacrés à la contestation.
} 


\begin{tabular}{c} 
Expertise ${ }^{4}$ \\
Partage d'information sur les pratiques professionnelles \\
Espace social \\
Espace contestataire \\
\hline
\end{tabular}

Ces critères ont été notés de 1 à 5 par deux experts spécialisés en systèmes d'information (analyse des données et interprétation des résultats). Les modalités de notation sont les suivantes :

- Structure du blog : nombre de catégories et d'archives appartenant soit à l'expertise, soit à la description des pratiques professionnelles, soit au social, soit à la contestation

- Page d'accueil du blog: analyse de la raison d'être du blog, ce qui a amené à sa création

- Analyse de contenu : sémantique et teneur des billets jusqu'au point de saturation de l'information

Figure 1 : L'analyse netnographique d'un blog

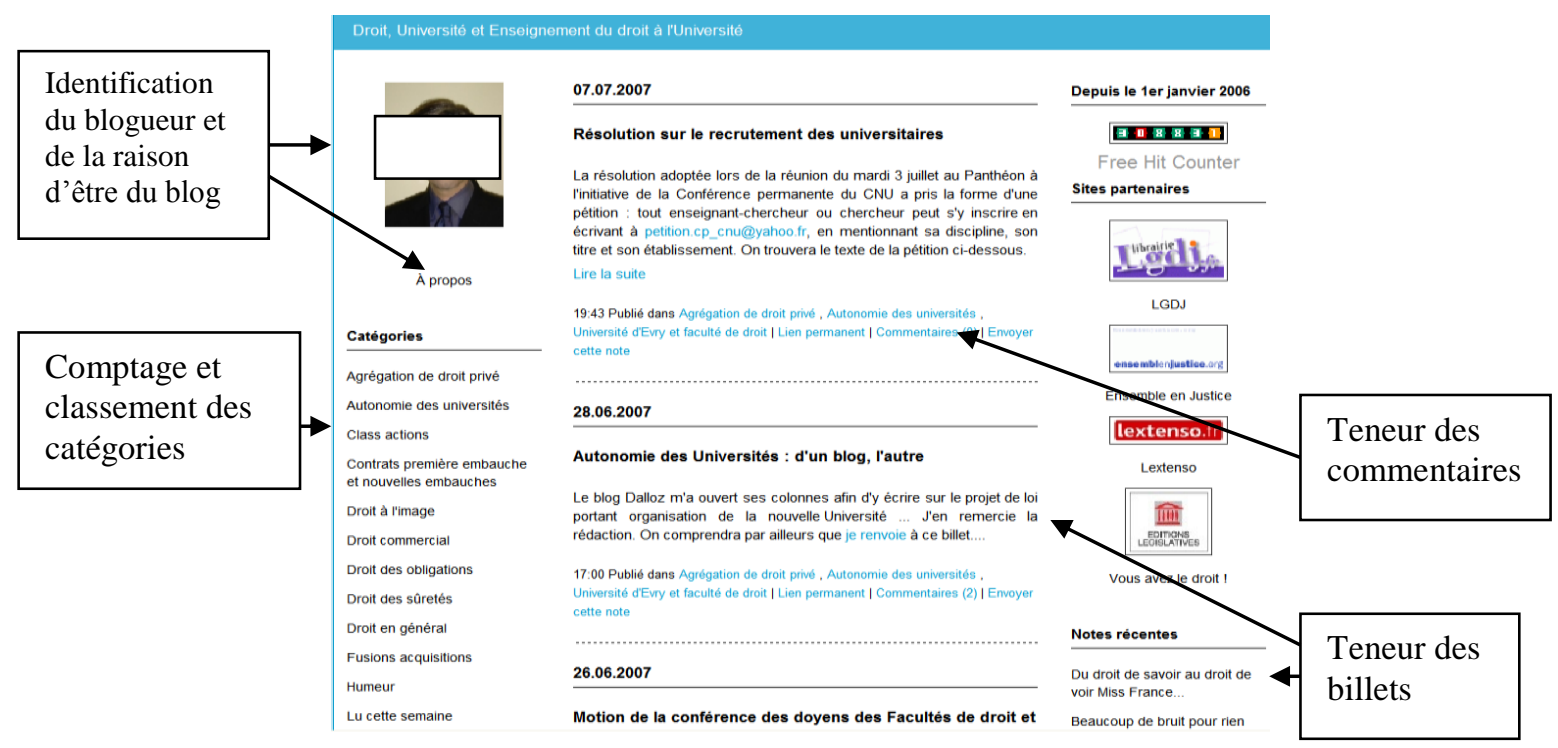

Les données ont ensuite été factorisées afin de déterminer les principales composantes pouvant servir de base à une typologie pertinente des blogs de salariés. L'analyse factorielle montre une bonne qualité de la représentation. La variance cumulée est de $85 \%$. La matrice des composantes fait apparaître deux composantes. Le premier axe factoriel oppose l'expertise au social et à la contestation. Le second axe correspond à l'intensité des pratiques professionnelles observées au sein des blogs.

Tableau 2 : résumé de l'analyse factorielle

\begin{tabular}{|c|c|c|c|}
\hline \multicolumn{2}{|c|}{ Communautés } & & Pourcentage de \\
\hline Initial & Extraction & Composants & \\
\hline
\end{tabular}

\footnotetext{
${ }^{4}$ L'expertise a été considérée au sein du référentiel métier. Ainsi, les informations juridiques liées à la formation ou au contrat de travail présentes sur les blogs des syndicats sont considérées comme des expertises.

${ }^{5}$ L'espace social correspond à l'ensemble des actions visant à renforcer l'esprit de corps des membres d'une même profession, entreprise ou activité. Il vise à développer la communication entre membres et l'entraide. Nous citerons les initiatives d'Affordance (le blog d'un maître de conférences en systèmes d'information) qui référence les blogs des maîtres de conférences, met en ligne des dossiers de qualification, organise la circulation de l'information sur les postes à pourvoir.
} 


\begin{tabular}{|l|l|l|l|l|l|}
\hline Expertises & 1 & 0,824 & $\begin{array}{l}1 / \\
\text { Expertise } \\
\text { Social } \\
\text { Contestation }\end{array}$ & $\begin{array}{l}-0,906 \\
0,824 \\
0,933\end{array}$ & 58,9 \\
\hline $\begin{array}{l}\text { Pratiques } \\
\text { professionnelles }\end{array}$ & 1 & 0,972 & $\begin{array}{l}\text { 2/ } \\
\text { Pratiques } \\
\text { professionnelles }\end{array}$ & 0,986 & 85,1 \\
\hline Social & 1 & 0,715 & KMO : 0,633 & \\
\hline Contestation & 1 & 0,896 & & \\
\hline
\end{tabular}

Figure 2 : Typologie des blogs de salariés

TYPOLOGIE DES BLOGS DE SALARIES

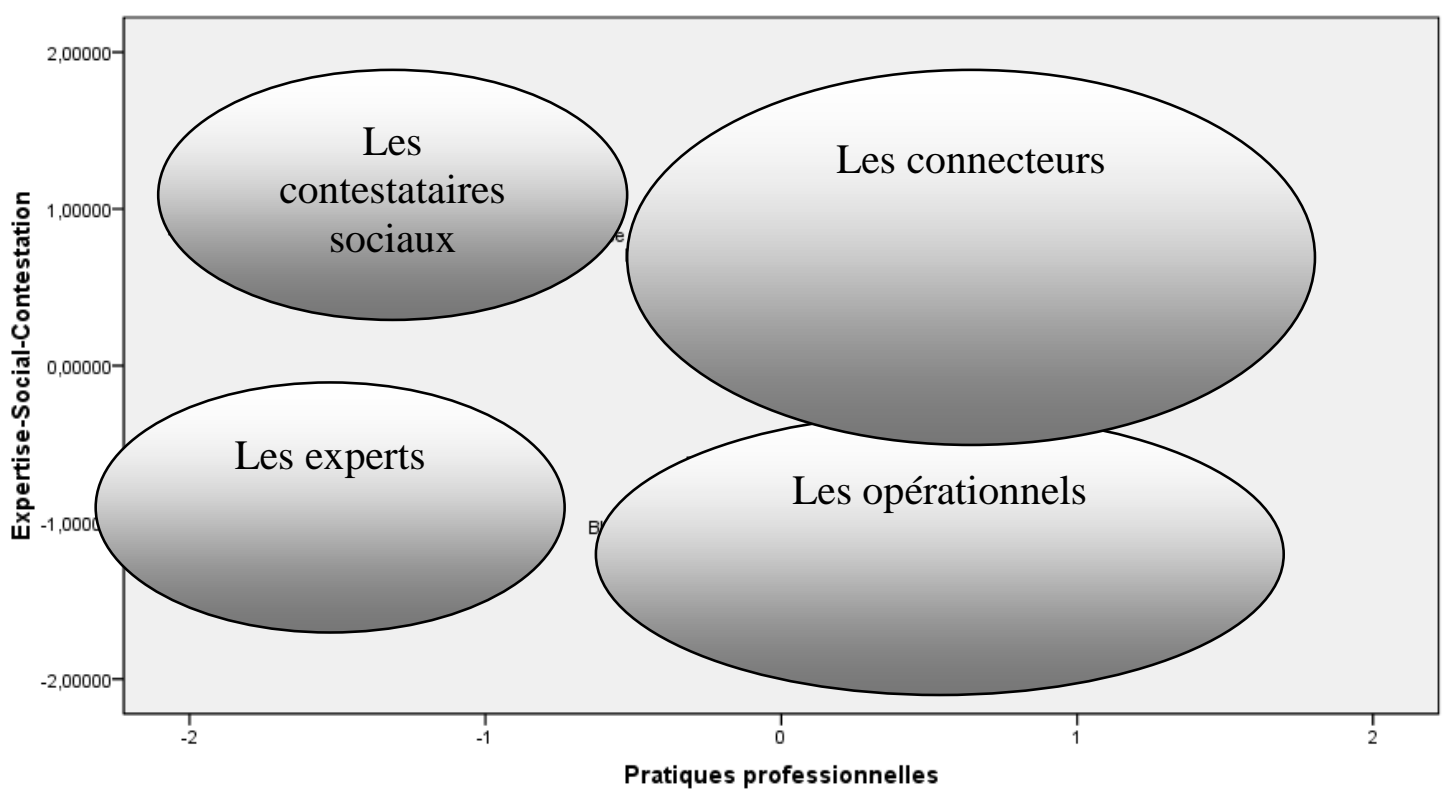

\subsection{Les opérationnels, une vision «métier» fondée sur le partage des pratiques}

La typologie obtenue est constituée de quatre catégories que nous avons nommées : experts, opérationnels, connecteurs, contestataires sociaux (figure 2).

Les blogs d'experts ont un double objectif. Pour les entreprises qui les sponsorisent, ces blogs leur donnent une image de technicité et de crédibilité. Les experts, quant à eux, accèdent à une notoriété et légitimité professionnelles fortes6. La croissance des blogs d'expert amène la blogosphère à s'interroger sur le rôle des communautés experts au sein des entreprises et sur les principes de leur fonctionnement.

Les contestataires sociaux sont essentiellement des salariés isolés anonymes. Leur contestation va de la contestation de l'entreprise d'une façon générale à une contestation plus personnelle de la façon dont ils sont managés au quotidien.

\footnotetext{
${ }^{6}$ Nous citerons le cas de Robert Scoble, blogueur star de Microsoft qui se définit comme un évangéliste technique (http://scobleizer.wordpress.com).
} 
Les connecteurs sont des blogs dont la spécificité est qu'ils constituent des espaces ouverts de discussion (forums pratiquement systématiques). Ils peuvent être créés soit par des acteurs sociaux bien identifiés, soit par les salariés directement dans une démarche spontanée.

La dernière catégorie, celle des opérationnels, est, à notre avis, celle qui présente le plus d'intérêt pour l'étude prospective des métiers.

Les opérationnels correspondent à la catégorie des blogs métiers ou entreprises dont l'objectif principal est la diffusion et le partage des meilleures pratiques. Ils permettent un regroupement de communautés professionnelles interentreprises avec comme socle commun le même secteur d'activité ou le même métier. L'échange par intérêt sur un sujet ou problématique amène une dynamique permettant l'émergence de standards de qualité, de sources d'innovation, de pratiques inédites ou au contraire usuelles. Il facilite également la formation de nouveaux salariés. La confiance apparaît comme un élément déterminant et fédérateur de ce type de communautés. Cette dernière se cristallise soit sur le créateur du blog (est-il légitime pour tenir ce blog ?), soit sur l'organisation (entreprise leader du marché, journal professionnel reconnu) initiatrice et modératrice de ce blog. 
Tableau 3 : Points de vue des blogueurs sur l'intelligence collective et le partage des pratiques à travers les blogs de salariés

\begin{tabular}{|c|c|}
\hline Verbatims & Thématiques \\
\hline $\begin{array}{l}\text { Aux deux piliers actuels de l'organisation d'entreprise -les hiérarchies fonctionnelles et les équipes projet-, il faudra ajouter un } \\
\text { troisième -les communautés - qui donnera aux entreprise les moyens d'une nouvelle croissance. Les entreprises qui survivront } \\
\text { au 21e siècle seront celles qui auront su mettre en œuvre les systèmes d'apprentissage les plus efficaces sur leur cœur de métier, } \\
\text { et qui auront externalisé tout le reste (Organisation } 2.0,2008 \text { ). }\end{array}$ & $\begin{array}{l}\text { Les } \\
\text { d'apprentissage : } \begin{array}{r}\text { activité } \\
\text { stratégique }\end{array}\end{array}$ \\
\hline $\begin{array}{l}\text { Le blog se situe davantage au niveau opérationnel, dans la transversalité ; passant outre les territoires internes et les guerres de } \\
\text { chapelle (Caspeex, 2006). }\end{array}$ & $\begin{array}{l}\text { Le décloisonnement des } \\
\text { idées et des pratiques }\end{array}$ \\
\hline $\begin{array}{l}\text { Le travail de veille se fait très facilement avec un blog. Chacun y poste le fruit de ses recherches et peut commenter les } \\
\text { résultats postés par d'autres collaborateurs. Ainsi se crée une dynamique d'échange et une mutualisation des idées innovantes. } \\
\text { Cela devient vite une surenchère d'idées, de trouvailles, et de bonnes pratiques au service de la collectivité (Caspeex, 2006). }\end{array}$ & $\begin{array}{l}\text { La motivation des salariés } \\
\text { autour de la production } \\
\text { d'idées }\end{array}$ \\
\hline $\begin{array}{l}\text { Il faut effectivement prendre en compte le temps de collaboration dans le management ! Je pense que le travail collaboratif et la } \\
\text { gestion des connaissances sont liés dans l'émergence de l'entreprise } 2.0 \text {. En effet la collaboration, comme toute nouvelle } \\
\text { organisation de travail, a besoin de compétences spécifiques (à prendre en compte dans les profils de poste). Elle est aussi } \\
\text { créatrice de sens (donc de connaissances). L'entreprise, si elle fait le choix des communautés de pratiques par exemple, devra } \\
\text { capitaliser pour pérenniser cette intelligence collective émergente. Reste également à manager cette intelligence (Froge Tech } \\
\text { dans Zazibao, 2008). }\end{array}$ & $\begin{array}{l}\text { Le management de } \\
\text { l'intelligence collective }\end{array}$ \\
\hline $\begin{array}{l}\text { Mais, même dans la perspective de l'ingénierie des connaissances, dont les travaux les plus intéressants sont les standards du } \\
\text { Web Sémantique, la question de la participation devra se poser. Et elle se pose même immédiatement à travers la notion de } \\
\text { confiance. Ce n'est en effet pas un hasard si, au sommet des briques du Web Sémantique, la dernière brique, véritable clef de }\end{array}$ & $\begin{array}{l}\text { La confiance antécédent de } \\
\text { la participation }\end{array}$ \\
\hline
\end{tabular}




\section{Les blogs de salariés : outil de prospective, une étude de cas menée sur les nouveaux métiers du Web}

\subsection{Description de la démarche de recherche}

Les opérationnels décrivent le présent et le futur de leur métier. Ils peuvent être identifiés aux acteurs-experts tels qu'ils sont décrits par Boyer et Scouarnec (2008). Au sein des opérationnels, il nous est apparu qu'un nombre important de métiers en émergence trouvait grâce aux blogs une plate-forme de diffusion et d'entraide. L'aspect pédagogique de ces blogs et leur vocation essentiellement tournée sur le métier (descriptif, évolutions, problématiques de formation, constitutions de réseaux) en font un matériau particulièrement approprié à l'étude de la prospective des métiers. Leur récence induit une acuité particulière à la projection vers l'avenir. Parmi ces nouveaux métiers, les métiers du Web occupent une place prépondérante adaptée au medium étudié à savoir Internet. Leur pratique du Web rend le recours au témoignage, au carnet de bord introspectif, aux interrogations sur leur métier particulièrement fécond et naturel. Notre démarche a donc été d'étudier la façon dont la prospective métier est pratiquée au sein de ces nouveaux métiers.

Dans un premier temps, nous avons effectué une taxinomie de ces métiers en fonction des compétences mobilisées. Par ailleurs, nous avons établi une distinction entre les différents types d'organisations de l'échantillon (organisations d'appartenance des blogueurs). L'objectif était de définir l'existant (le présent) le plus précisément possible.

Dans un second temps, en nous fondant sur les outils de prospective développés selon la méthode des scénarios, nous avons analysé et confronté l'existant à la projection des acteurs à savoir les mutations internes et externes auxquels ceux-ci pensent être confrontés à l'avenir. En outre, nous avons distingué un certain nombre de facteurs environnementaux (politique, formation, comportements) que nous avons dénommé zones d'incertitude. Les zones d'incertitude sont constituées de thématiques en débat au sein de la blogosphère. Le croisement des variables internes et externes nous a permis d'élaborer un certain nombre de scénarios regroupés selon plusieurs thématiques. Le choix des thématiques et l'élaboration des scénarios a été effectué au sein d'un groupe restreint de professionnels correspondant aux familles déterminées à partir de la typologie des différents métiers.

\section{Figure 3 : Méthodologie suivie par le chercheur pour l'élaboration des scénarios} prospectifs

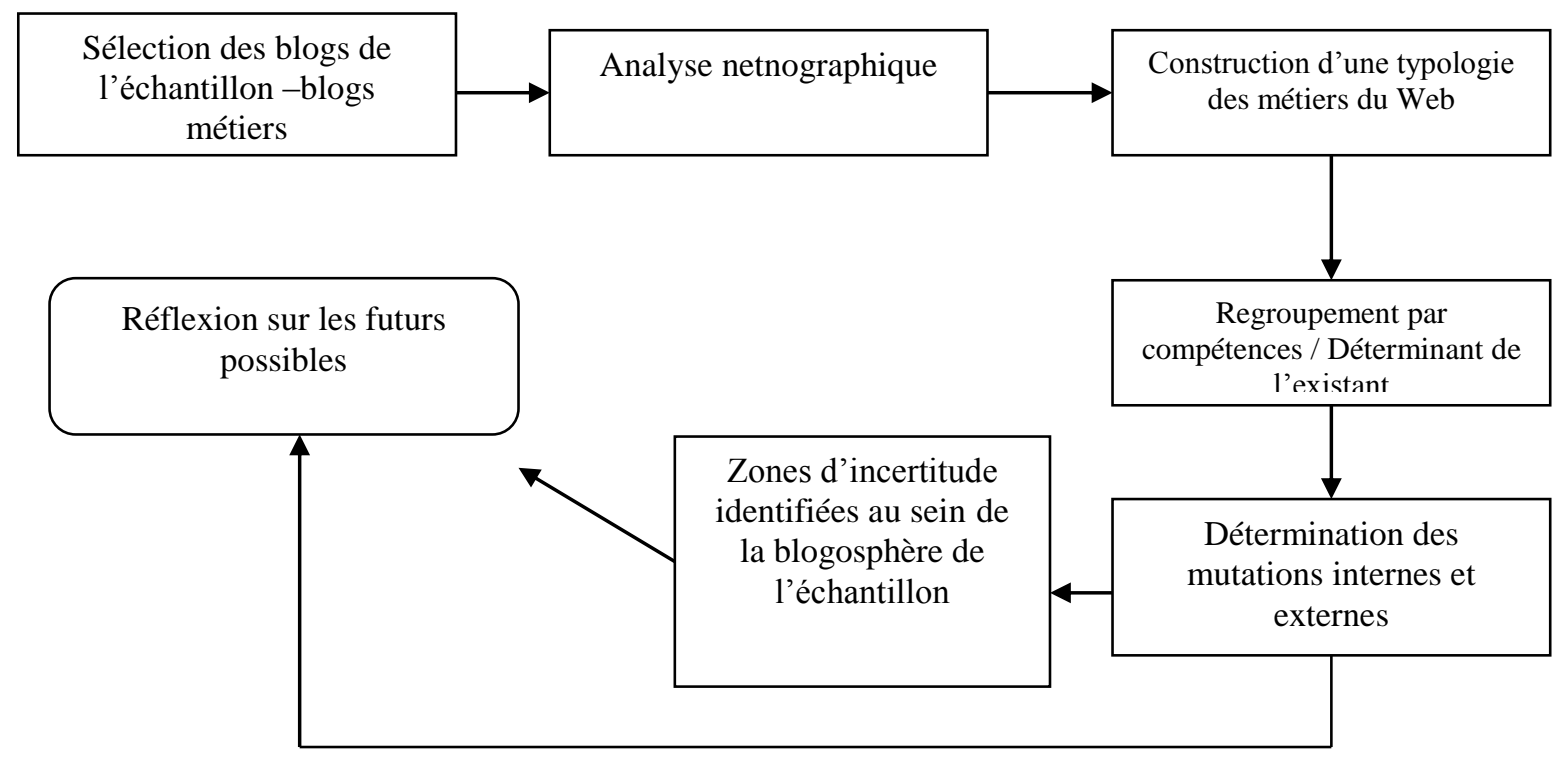




\subsection{Présentation des nouveaux métiers du Web, une illustration du concept de « nouveau métier » défini par les salariés}

Nous considérerons que les nouveaux métiers du Web sont des métiers en émergence dont la plupart n'a pas de fiche métier détaillée et de référentiel arrêté. Le caractère «mouvant» de ces métiers adapté à des pratiques professionnelles dépendantes des évolutions technologiques de l'Internet fait que la délimitation, la dénomination et la description des référentiels des métiers et des compétences sont particulièrement difficiles à mettre en place.

Notre définition d'un nouveau métier est volontairement large. Au contraire de Tissioui (2008), nous n'établissons pas de distinction entre nouveau métier et métier en émergence. Par ailleurs, nous incluons dans notre corpus de nouveaux métiers des métiers anciens en transformation du fait de l'adjonction de nouvelles compétences. Premièrement, il nous semble délicat d'effectuer une distinction entre métier en émergence (selon la définition de Tissioui (2008), un métier dont les prémisses d'activité existent de manière éclatée) et nouveau métier (récemment reconnu). Deuxièmement, l'adjonction de compétences nouvelles à un métier ancien l'amène à le transformer souvent radicalement. Le flou conceptuel entourant la notion même de nouveau métier (où s'arrête l'émergence, où commence l'existence légale et reconnue d'un métier ?) est relevée par la blogosphère elle-même. La blogosphère liée à ces métiers s'est organisée afin d'offrir au marché une vision la plus exacte possible de leur profil. A partir de certains billets comme celui du blog «profession.Web.ch » de janvier 2006, les blogueurs francophones (France, Belgique, Suisse essentiellement) ont ouvert à travers des commentaires et des trackbacks des discussions libres sur leurs métiers (descriptions, fonctions, formations...). Cette discussion est encore en cours en 2009 avec l'ajout presque quotidien de nouveaux métiers identifiés.

Cette démarche tirée des blogs de salariés a été reprise par les institutions dont l'APEC qui a mis à disposition des internautes un $\mathrm{Wiki}^{7}$ permettant aux salariés de remplir eux-mêmes leur fiche métier (finalités, missions; rémunérations, compétences, profils...), des espaces de discussion associés et des modifications en ligne possibles ainsi qu'un historique de ces modifications. Une analyse faite à partir d'une centaine de blogs classés selon la typologie présentée précédemment a permis d'établir une liste la plus exhaustive possible de ces métiers ainsi qu'une classification selon le type d'expertise nécessaire. Les blogs d'opérationnels sont majoritaires dans l'échantillon mais un certain nombre de blogs d'experts et de connecteurs a été intégré dans l'analyse du fait de l'apport d'information supplémentaire apporté.

A partir de cette analyse, plus d'une soixantaine de métiers a été répertoriée. Ils ont la particularité d'être à la fois des métiers techniques et liés à la communication. Certains sont des adaptations des métiers de la communication issus des médias et agences traditionnels. D'autres sont des métiers radicalement nouveaux issus du Web 2.0 et de sa version 3.0 dite Web sémantique. Enfin, on notera l'émergence de métiers issus des activités de gestion documentaire et gestion de la connaissance (tableau 4). Un regroupement des métiers a été effectué selon les domaines de compétences auxquels ils appartiennent. Le regroupement s'est effectué à partir de onze domaines de compétences. Les onze domaines de compétences retenues l'ont été sur trois critères permettant d'estimer les savoirs et savoirs faire de chaque métier :

Le profil des blogueurs (formation, expérience antérieure)

Les descriptifs métiers recueillis au sein des blogs et des sites institutionnels

Les témoignages (parcours)

\footnotetext{
${ }^{7}$ Système de gestion de contenus en libre accès et fondé sur une écriture collaborative
} 
Figure 4 : Démarche de recherche visant à établir une classification des métiers du Web

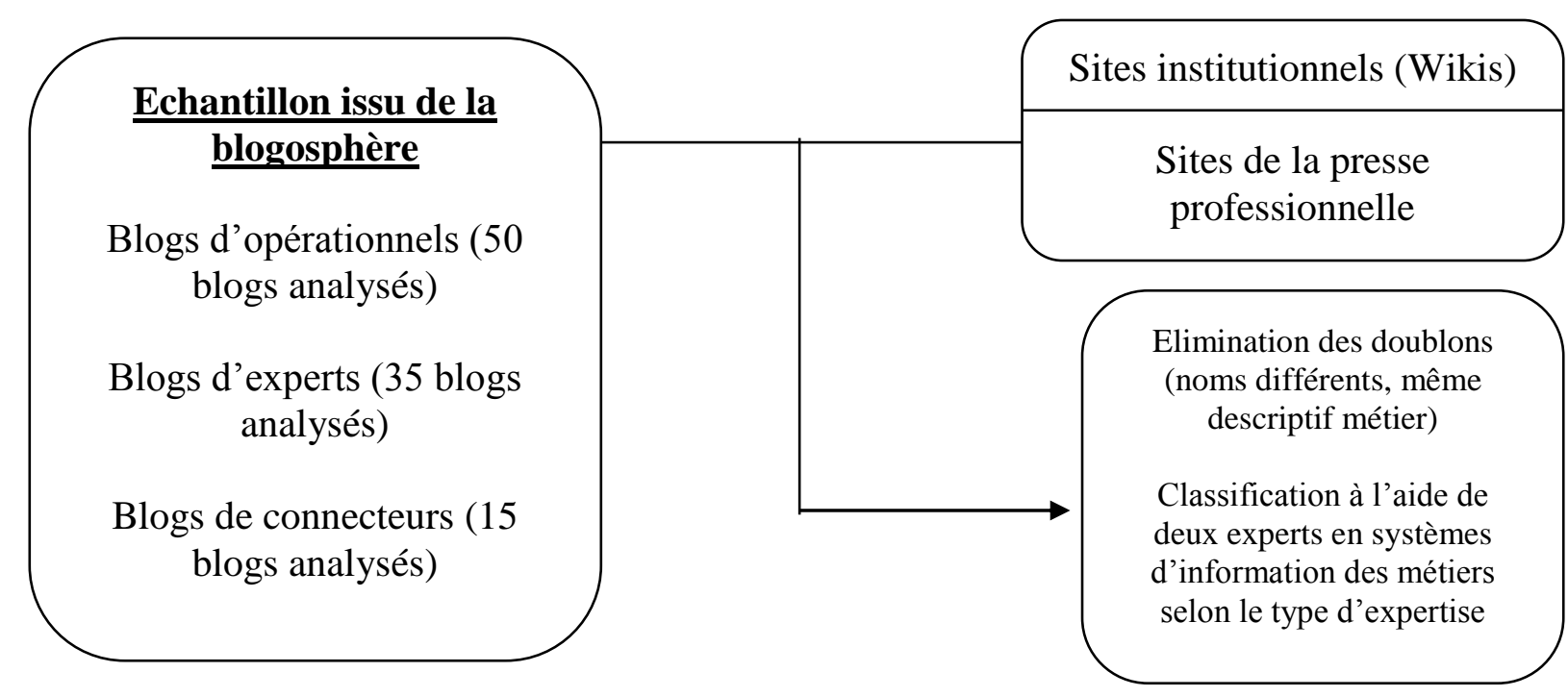

En fonction des regroupements de métiers effectués, nous nous sommes attachés à définir l'existant de ces derniers selon les critères définis par Berard (2002) et tirés d'une étude action sur le thème «processus d'introduction et impact des TIC dans les organisations » à partir d'une soixantaine d'études de cas réalisée par l'ANACT ${ }^{8}$. Cette grille a été complétée par l'ensemble des observations obtenues par notre recherche. Les observations pertinentes sur le sujet (plus de 200) ont été préalablement codées puis regroupées sur la base des cinq thématiques de la grille (tableau 5).

Le recensement des facteurs de changement du domaine (tableau 6) a permis de distinguer :

- Les facteurs «formation et démographie »

- Les facteurs politiques

- Les facteurs internes au marché de l'Internet (économie du Web, comportement du consommateur, nouveaux standards)

- Les zones d'incertitude qui font débat au sein de la blogosphère

\footnotetext{
${ }^{8}$ Agence nationale pour l'amélioration des conditions de travail
} 
Tableau 4 : Les principaux métiers du Web identifiés au sein de la blogosphère selon le type de compétence

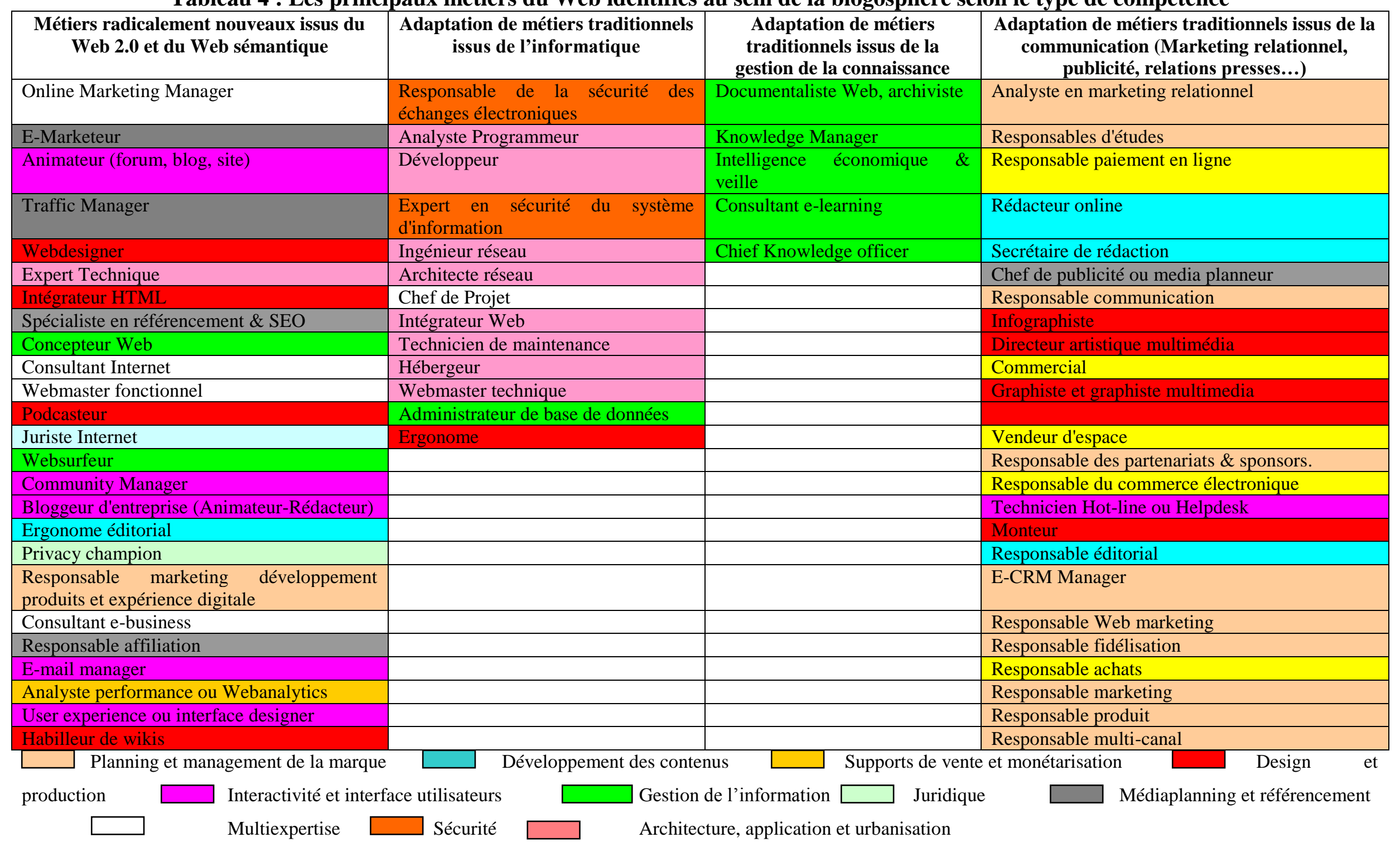


Tableau 5 : Détermination de l'existant par application de la grille d'analyse de l'ANACT « conduite des projets TIC : usages, acteurs », thématiques émergentes des blogs de l'échantillon

\begin{tabular}{|c|c|c|c|c|c|}
\hline Contexte organisationnel & Management & $\begin{array}{l}\text { Compétences } \\
\text { concepteurs }\end{array}$ & Représentation métier & Nature des outils TIC & $\begin{array}{l}\text { Nature des relations entre les } \\
\text { concepteurs et les utilisateurs }\end{array}$ \\
\hline $\begin{array}{l}\text { Organisations } \\
\text { "achètent à l'extérieur » } \\
\text { des structures compétentes } \\
\text { en acquisition et traitement } \\
\text { de l'information } \\
\text { Grandes agences de } \\
\text { publicité, annonceurs }\end{array}$ & $\begin{array}{l}\text { Hiérarchie } \\
\text { «descendante » de } \\
\text { l'information, structures } \\
\text { verticales mutant vers } \\
\text { l'horizontalité (gestion } \\
\text { de projets) }\end{array}$ & $\begin{array}{l}\text { Très diverses et } \\
\text { souvent issues de } \\
\text { mobilité } \\
\text { professionnelle forte } \\
\text { permettant } \\
\text { l'acquisition } \\
\text { d'expériences } \\
\text { pointues. }\end{array}$ & $\begin{array}{l}\text { Perception fondée sur les } \\
\text { usages voulus ou } \\
\text { souhaités par les } \\
\text { utilisateurs. L'expertise } \\
\text { au service des non- } \\
\text { experts de l'entreprise. }\end{array}$ & $\begin{array}{l}\text { Les outils s'inscrivent } \\
\text { dans une perspective } \\
\text { d'évolution des méthodes } \\
\text { de travail ainsi que par la } \\
\text { recherche d'une meilleure } \\
\text { productivité des actions } \\
\text { marketing et de } \\
\text { communication. }\end{array}$ & $\begin{array}{lr}\text { Réactivité des experts } & \text { aux } \\
\text { demandes des non-experts. }\end{array}$ \\
\hline $\begin{array}{l}\text { Organisations spécialisées } \\
\text { dans la sécurisation de } \\
\text { l'information } \\
\text { SSII }\end{array}$ & $\begin{array}{l}\text { Orientation mise sur la } \\
\text { formation et l'évolution } \\
\text { des personnels, stress } \\
\text { important des personnels }\end{array}$ & $\begin{array}{l}\text { Purement techniques } \\
\text { en début de carrière } \\
\text { puis commerciales et } \\
\text { marketing avec } \\
\text { fonctions } \\
\text { d'animation et de } \\
\text { gestion d'équipes }\end{array}$ & $\begin{array}{l}\text { Perception fondée sur } \\
\text { l'accompagnement et la } \\
\text { formation des utilisateurs } \\
\text { ainsi que la } \\
\text { compréhension des } \\
\text { informations émanent des } \\
\text { utilisateurs afin d'adapter } \\
\text { au mieux l'offre. }\end{array}$ & $\begin{array}{l}\text { Les outils s'inscrivent } \\
\text { dans une perspective } \\
\text { stratégique } \\
\text { organisationnelle et vont } \\
\text { (au-delà de la sécurité des } \\
\text { réseaux) du conseil expert } \\
\text { à l'optimisation des } \\
\text { processus métier de } \\
\text { l'entreprise conseillée. }\end{array}$ & $\begin{array}{ll}\text { Relation marchande entre } \\
\text { concepteurs et utilisateurs }\end{array}$ \\
\hline $\begin{array}{l}\text { Organisations } \\
\text { traditionnelles ou la fracture } \\
\text { numérique peut être } \\
\text { importante } \\
\text { Bibliothèques }\end{array}$ & $\begin{array}{l}\text { Diversité des modes de } \\
\text { management en fonction } \\
\text { des organisations et de la } \\
\text { culture d'entreprise }\end{array}$ & $\begin{array}{l}\text { Variables mais en } \\
\text { évolution afin de } \\
\text { répondre } \\
\text { nouveaux besoins de } \\
\text { l'information } \\
\text { documentaire en } \\
\text { ligne et des nouvelles } \\
\text { stratégies r de } \\
\text { numérisation des } \\
\text { données }\end{array}$ & $\begin{array}{l}\text { Perception par les acteurs } \\
\text { d'un métier révolutionné } \\
\text { par les TIC. Forte prise } \\
\text { de conscience de } \\
\text { l'obligation de muter vers } \\
\text { le numérique et les } \\
\text { plates-formes } \\
\text { collaboratives. }\end{array}$ & $\begin{array}{l}\text { Les outils s'inscrivent } \\
\text { dans une perspective de } \\
\text { modification et re- } \\
\text { traitement voire de re- } \\
\text { documentation } \\
\text { données des } \\
\text { indexées, cataloguées, } \\
\text { découpées. Ils sont } \\
\text { parfois perçus comme une } \\
\text { dénaturation du livre. }\end{array}$ & $\begin{array}{l}\text { Relation fondée sur la formation } \\
\text { continue jugée intensive et } \\
\text { spécifique à leur secteur par les } \\
\text { blogueurs. }\end{array}$ \\
\hline
\end{tabular}




\begin{tabular}{|c|c|c|c|c|c|}
\hline $\begin{array}{l}\text { Organisations qui font du } \\
\text { partage d'information une } \\
\text { compétence à valoriser sur } \\
\text { le marché de la } \\
\text { connaissance } \\
\text { Cabinets de conseil, } \\
\text { consultants e-learning, } \\
\text { agences en marketing } \\
\text { relationnel, experts en data- } \\
\text { mining, }\end{array}$ & $\begin{array}{l}\text { Management participatif, } \\
\text { structures en réseau }\end{array}$ & $\begin{array}{l}\text { Adeptes de l'auto- } \\
\text { formation } \\
\text { notamment via } \\
\text { Internet. } \\
\text { Appréciation forte de } \\
\text { la polyvalence. }\end{array}$ & $\begin{array}{l}\text { Représentation fondée sur } \\
\text { la notion de concepts } \\
\text { alternatifs, et d'artisans } \\
\text { créatifs. Passages en free- } \\
\text { lance fréquents, effets } \\
\text { longue traîne où chacun } \\
\text { peut être son propre } \\
\text { entrepreneur. }\end{array}$ & $\begin{array}{l}\text { Les outils sont } \\
\text { secondaires dans la } \\
\text { démarche adoptée. Leur } \\
\text { mise en avant est toujours } \\
\text { justifiée par leur capacité } \\
\text { de service et leur valeur } \\
\text { d'usage. }\end{array}$ & $\begin{array}{l}\text { Relation marchande entre } \\
\text { concepteurs et utilisateurs. } \\
\text { Démarche de fidélisation des } \\
\text { utilisateurs / clients. }\end{array}$ \\
\hline $\begin{array}{l}\text { Organisations qui mettent } \\
\text { en forme l'information, la } \\
\text { valorisent et la } \\
\text { commercialisent } \\
\text { Editeurs de sites Web, } \\
\text { Brokers en bases de } \\
\text { données, moteurs de } \\
\text { recherche, micro- } \\
\text { entrepreneurs }\end{array}$ & $\begin{array}{l}\text { Autonomie des } \\
\text { utilisateurs pour faire } \\
\text { évoluer les méthodes de } \\
\text { travail, fort } \\
\text { individualisme des } \\
\text { stratégies de carrières }\end{array}$ & $\begin{array}{l}\text { Formation du } \\
\text { personnel aux } \\
\text { applications } \\
\text { collaboratives } \\
\text { Optimisation des } \\
\text { processus métier, } \\
\text { forte polyvalence } \\
\text { pour certains } \\
\text { Expertises en } \\
\text { mutation } \\
\text { permanentes }\end{array}$ & $\begin{array}{l}\text { Représentation } \\
\text { «projective » des métiers } \\
\text { avec une forte dimension } \\
\text { essai-erreur. }\end{array}$ & $\begin{array}{l}\text { Les outils sont en lien } \\
\text { avec cet état d'esprit } \\
\text { «projectif». Lancement } \\
\text { permanent de versions } \\
\text { Bêta afin d'en valider les } \\
\text { performances auprès des } \\
\text { utilisateurs. Mise en } \\
\text { versions libres d'outils à } \\
\text { destination } \\
\text { internautes, fort esprit } \\
\text { open-source } \\
\text { certains. }\end{array}$ & $\begin{array}{l}\text { Relation marchande et non } \\
\text { marchande orientée services, } \\
\text { mission «d'évangélisation } \\
\text { technique » (blogs, animation } \\
\text { des wikis, campagnes de } \\
\text { communication à destination de } \\
\text { certaines cibles). }\end{array}$ \\
\hline
\end{tabular}


Tableau 6 : Recensement des facteurs de changement du domaine, thématiques émergentes des blogs de l'échantillon

\begin{tabular}{|c|c|c|c|}
\hline $\begin{array}{l}\text { Mutations externes } \\
\text { Pratiques pédagogiques } \\
\text { et de formations, } \\
\text { démographie }\end{array}$ & $\begin{array}{l}\frac{\text { Mutations }}{\text { externes }} \\
\text { Contexte } \\
\text { politique }\end{array}$ & Mutations internes & $\frac{\text { Mutations externes et mutations internes }}{\text { Zones d'incertitudes }}$ \\
\hline \multirow{7}{*}{$\begin{array}{l}\text { Evolution de l'approche } \\
\text { pédagogique : alternance, } \\
\text { auto-formation, } \\
\text { formation par imitation } \\
\text { des bonnes pratiques }\end{array}$} & \multirow{2}{*}{$\begin{array}{l}\text { Soutien des } \\
\text { pouvoirs publics } \\
\text { aux NTIC }\end{array}$} & \multirow{6}{*}{$\begin{array}{c}\text { Essor de la publicité en ligne et du } \\
\text { ciblage comportemental } \\
\text { Diminution des coûts liés à la } \\
\text { communication institutionnelle sur } \\
\text { Internet } \\
\text { Augmentation des études marketing en } \\
\text { ligne } \\
\text { Croissance régulière du commerce en } \\
\text { ligne } \\
\text { Emergence du C2C (consumer to } \\
\text { consumer) }\end{array}$} & $\begin{array}{l}\text { Menaces sur les formations courtes plébiscitées par le marché (réforme du } \\
\text { financement des IUT) }\end{array}$ \\
\hline & & & $\begin{array}{c}\text { Démocratisation des applicatifs (mashups, API) permettant le développement du } \\
\text { « contenu généré par l'ensemble des utilisateurs » }\end{array}$ \\
\hline & \multirow{5}{*}{$\begin{array}{c}\text { Implication des } \\
\text { organisations } \\
\text { internationales } \\
\text { dans la société } \\
\text { de l'information } \\
\text { et sa } \\
\text { globalisation } \\
\text { (OMC, FMI, } \\
\text { Banque } \\
\text { Mondiale) }\end{array}$} & & Essai de récupération des API par certains fournisseurs \\
\hline & & & Fracture numérique en réduction \\
\hline & & & Limitation de la mondialisation des NTIC sur les pays en voie de développement \\
\hline & & & $\begin{array}{l}\text { Développement de la gratuité (open-source, logiciels libres) et des moyens de } \\
\text { production par les utilisateurs eux-mêmes }\end{array}$ \\
\hline & & $\begin{array}{l}\text { Diffusion des technologies liées au } \\
\text { Knowledge management }\end{array}$ & $\begin{array}{l}\text { Limitations des téléchargements libres, guérilla open-source et institutionnels } \\
\text { (Google, Microsoft) }\end{array}$ \\
\hline \multirow{5}{*}{$\begin{array}{l}\text { Manque de formations } \\
\text { «pointues » adaptées aux } \\
\text { nouveaux métiers du } \\
\text { Web } \\
\text { Externalisation de } \\
\text { certaines compétences } \\
\text { (développeurs en Inde) }\end{array}$} & \multirow{5}{*}{$\begin{array}{l}\text { Démocratisation } \\
\text { de l'ADSL et } \\
\text { des nouveaux } \\
\text { formats } \\
\text { numériques } \\
\text { Développement } \\
\text { des plans } \\
\text { numériques } \\
\text { nationaux }\end{array}$} & \multirow[t]{2}{*}{ Accroissement des pratiques de e-GRH } & Surveillance et gouvernance de l'Internet par les gouvernements \\
\hline & & & \multirow[t]{2}{*}{$\begin{array}{l}\text { Fin de la neutralité des réseaux du fait d'une augmentation des coûts d'accès } \\
\text { imposés par les fournisseurs de contenus et d'applications }\end{array}$} \\
\hline & & \multirow{3}{*}{$\begin{array}{c}\text { Interopérabilité technique, sémantique, } \\
\text { organisationnelle } \\
\text { Interactivité croissante (technologie Ajax) }\end{array}$} & \\
\hline & & & $\begin{array}{l}\text { Apparition de nouveaux acteurs (notamment des moteurs de recherche) grâce au } \\
\text { Web sémantique }\end{array}$ \\
\hline & & & \multirow[t]{2}{*}{ Diffusion lente du Web sémantique (format RDF encore peu présent) } \\
\hline \multirow{3}{*}{$\begin{array}{l}\text { Montée en puissance des } \\
\text { «Digital Natives » }\end{array}$} & \multirow{3}{*}{$\begin{array}{l}\text { Taxation des } \\
\text { FAI } \\
\text { Imposition des } \\
\text { micro-revenus } \\
\text { générés sur le } \\
\text { Web }\end{array}$} & \multirow{2}{*}{$\begin{array}{l}\text { Reconnaissance de l'importance des } \\
\text { réseaux sociaux }\end{array}$} & \\
\hline & & & \multirow[t]{2}{*}{ Apparition des nouveaux influenceurs en ligne (blogueurs, consommateurs) } \\
\hline & & $\begin{array}{c}\text { Fragmentation des médias au profit des } \\
\text { médias mobiles et alternatifs } \\
\text { Convergence des médias traditionnels et } \\
\text { d'Internet }\end{array}$ & \\
\hline
\end{tabular}




\subsection{Présentation des scénarios prospectifs}

La présentation des différentes données du cas à notre groupe d'experts a permis de définir un certain nombre d'axes prospectifs ou hypothèses :

- L'hypothèse de la résistance qui s'assimile à la résistance au changement soit de la part des entreprises, soit de la part des consommateurs, soit de la part des pouvoirs publics

- L'hypothèse de la normalisation qui va dans le sens de la convergence et de la standardisation des référentiels et des pratiques

- L'hypothèse de l'éclatement qui imagine l'introduction dans les organisations des nouveaux métiers du Web comme un «virus » détruisant peu à peu leurs structures d'accueil

- L'hypothèse de l'enrichissement dans laquelle les mutations s'effectuent en douceur au sein de mécaniques gagnant-gagnant

\section{L’hypothèse de la résistance}

Le scénario de la démonétarisation : l'internaute personnalise à l'extrême sa page d'accueil, installe systématiquement des mashups de protection à l'invasion publicitaire ${ }^{9}$, créera son «desktop » et ses «widgets » adaptés. Il diffuse l'esprit de l'open-source entraînant une démonétarisation des métiers du web et une « déprofessionnalisation » des acteurs.

Le scénario de la résistance des organisations : 1'entreprise 2.0 puis sémantique reste un concept purement théorique. Les organisations recadrent dans leur fonctionnement traditionnel l'esprit du Web porté par les nouveaux métiers. Elles appliquent aux outils collaboratifs et à leur «nouveau» personnel un ROI (retour sur investissement) inadapté fondé sur le court terme et des leviers facilement maîtrisables. Les professionnels de ces métiers sont rejetés à la périphérie des organisations voire externalisés.

Le scénario du retour des Etats : effrayés par 1' espace contestataire que constitue le Web, saisi d'un certain nombre d'interrogations concernant les techniques de ciblage et leur caractère intrusif, les Etats prennent des mesures coordonnées pour limiter la liberté d'expression sur le Web. Les législations restrictives minorent peu à peu l'intérêt des internautes. La diminution des audiences on-line a une influence sur la publicité et à terme menace l'ensemble de l'économie du Web dont ses nouveaux métiers.

\section{L'hypothèse de la normalisation}

Le scénario de la convergence technologique : les technologies au départ fondées sur des fonctionnalités distinctes vont évoluer vers des technologies qui se recouperont de plus en plus introduisant un brouillage de la notion même de métier. Tendance encore renforcée par l'interopérabilité des médias sociaux, des médias traditionnels, des outils et du collaboratif.

Le scénario de la standardisation : les professionnels du Web s'associent pour proposer des référentiels métiers stabilisés. L'objectif est de gagner en lisibilité à l'égard du marché et de visibilité à l'égard des recruteurs. Cette standardisation est fortement influencée par les grands

\footnotetext{
${ }^{9}$ Exemple, Doritos qui est une «killer application» qui remplace les publicités en ligne par les photos personnelles de l'internaute.
} 
de l'Internet qui servent de garant à ce processus. La fin de standards ouverts et évolutifs contribue à retarder l'adoption de certaines innovations.

Le scénario de la dissémination à marche forcée : les nouveaux métiers du Web profitent de la restructuration de l'ensemble du secteur de la communication (des agences de publicité au marché de l'information documentaire). Google et les nouveaux entrants du monde de l'Internet imposent leurs standards :

En terme de numérisation des documents (Google Book)

En terme de médiaplanning (Google Adsens)

En terme de ciblage comportemental

En terme d'affiliation

Ces nouveaux standards entraînent la disparition des métiers traditionnels comme celui de médiaplanneur et de bon nombre d'agences média. Le métier du conseil est également touché puisque les grands de l'Internet montent des structures de conseil en stratégie d'abord sur Internet puis avec une optique multi-canal touchant l'ensemble des médias. Ces derniers sont également condamnés à évoluer: médias alternatifs contre presse papier, podcasting, affichage numérique et interactif, télévision gratuite sur le Web...Les survivants voient leurs méthodes de production radicalement changer. Les DSI s'impliquent dans un processus de cocréation de valeur avec les métiers.

\section{L'hypothèse de l'éclatement}

L'hypothèse de l'éclatement peut se concevoir à plusieurs niveaux :

- Au niveau organisationnel

- Au niveau individuel

- Au niveau des normes et des standards

Au niveau organisationnel

Le scénario de la tour d'ivoire: les outils se complexifient et restent pilotés par les experts. Leur importation dans les entreprises se heurte à leur inadaptation à l'organisation. Le collaboratif souffre d'un manque général de participation qui amène peu à peu l'abandon des projets phares comme les portails intranet. Cette inadaptation est due également au manque d'implication des managers (fracture générationnelle) et à un changement de culture difficile à réaliser dans les DSI. Les connaissances métiers diffusées à travers de mini-sites sectoriels sont répertoriées sans lien collaboratif entre elles. Ces plates-formes à l'arborescence étendue servent de support à l'émergence de certaines personnalités expertes qui s'autonomisent de plus en plus par rapport à l'entreprise.

Le scénario de l'entreprise atomisée : la diffusion des nouveaux outils entraîne une remise en cause des méthodes de management. L'auto-organisation venant de la base aboutit à redéfinir l'entreprise comme une myriade de start-ups organisées en réseau. La notion de «supplychain informationnelle » devient déterminante. Les DRH échouent à comprendre et intégrer ces nouveaux métiers laissant aux opérationnels la mission d'intégrer les nouvelles compétences et de planifier les recrutements en fonction d'évolutions permanentes. Le collaboratif rend poreux les frontières de l'entreprise qui développe des partenariats ouverts et parfois non sécurisés (échecs des DSI ?) avec d'autres «mondes»: free-lances, réseaux informels, communautés épistémiques indépendantes. Les nouveaux outils permettent la nomadisation des équipes et le multitâche qui amène à un "décompartimentation » des fonctions. Ces petites structures érigent l'ahierarchie en premier principe de fonctionnement. 
Au niveau individuel

Le scénario de la guerre des talents (1): les dispositifs de formations professionnelles peinent à fournir des professionnels de ces nouveaux métiers. La pénurie notamment au niveau des développeurs s'accentue. L'auto-formation se développe. Les entreprises se disputent les rares compétences disponibles sur le marché. Cette tension sur le marché de l'emploi retarde les projets 2.0 et les mutations indispensables dictées par le poids croissant de l'Internet sur les modes de consommation.

Le scénario de la guerre des talents (2) : les nouveaux talents du Web remplacent l'autorité émanent de la hiérarchie par une autorité fondée sur la popularité. Le capital réseau supplante le capital social. Les diplômés sont concurrencés par ces nouveaux influenceurs qui tirent leur légitimité de réseaux extérieurs à l'entreprise (clients, presse, actionnaires).

Le scénario de la déstructuration du collectif: les nouveaux métiers font rentrer dans l'organisation des individus hypermobiles se percevant comme offreurs de services plus que salariés. Ils développent des stratégies individuelles de carrière et conservent au-delà de l'entreprise d'autres espaces de mise en avant de soi à travers leurs réseaux sociaux.

Le scénario du bouleversement des références temporelles, spatiales et identitaires : les outils asynchrones, la recherche permanente d'agilité et de réactivité, la nomadisation, la fin des frontières privées-professionnelles brouillent les identités, les rythmes de travail, les ancrages physiques (bureau virtuel). Les salariés des métiers traditionnels peinent à s'adapter et ne se reconnaissent plus dans une organisation déstructurée. Une scission se crée entre tenants des anciens modes de travail et partisans des nouveaux.

Le scénario de l'autonomie contrôlée : les nouveaux métiers du Web sont perçus comme mettant en place une organisation à la fois autonome mais également contrôlée. La «traçabilité »du salarié est rendue possible par le développement de certaines technologies initialement mises en place pour développer la collaboration. Les experts savent brouiller les pistes, les autres sont victimes d'une nouvelle fracture numérique fondée sur la capacité à savoir neutraliser ou non les outils de contrôle.

Au niveau des normes et des standards

Le scénario des référentiels mouvants : les référentiels métiers disparaissent du fait de la modification constante des outils qui amènent l'émergence rapide de nouvelles compétences. La notion même de compétence peut être remise en cause. Elle est remplacée par le concept d'assemblage de compétences distinctes.

\section{L'hypothèse de l'enrichissement mutuel}

Le scénario des métiers pont: les nouveaux métiers se regroupent en deux catégories, les métiers citadelles (pointus, refermés sur de petites communautés d'experts) et les métiers pont (polyvalents et regroupant de multiples compétences). Le développement des métiers pont (urbanistes, experts techniques) permet de «récupérer» des personnels qui voient mourir leurs métiers traditionnels. La reconversion est facilitée grâce à la conservation par ces métiers de certaines compétences traditionnelles. 
Le scénario du dialogue social collaboratif : les nouveaux métiers du Web se diffusent dans le monde syndical. L'ouverture de blogs synchronisés, la mobilisation via les Wikis, les flux RSS, les forums renforcent le pouvoir contestataire des salariés mais également renforce le dialogue social.

L'établissement de ces différents scénarios permet de faire émerger des tendances lourdes sur l'évolution de l'économie du Web et d'aborder un certain nombre de problématiques liées à l'intégration de nouveaux métiers fortement novateurs dans leur pratiques et façons d'aborder l'organisation au sens général du terme.

Notre objet de recherche nous a conduit à utiliser de manière originale une nouvelle méthode d'analyse de données initialement qualitative mais qui peut également être traitée de façon quantitative. Cette méthode conçue pour écouter le consommateur est adaptée à une écoute du salarié tant que celui-ci considère le Web comme un espace de liberté, de contact professionnel, de vente et de présentation de ses compétences.

\section{Discussion}

Les apports méthodologiques de cette recherche montrent que la netnographie est une voie possible d'analyse des pratiques professionnelles actuelles et futures. Néanmoins, elle doit s'accompagner d'une rigueur méthodologique afin de collecter les données correspondant réellement à l'objet de recherche et interpréter celles-ci de manière objective. Comme le souligne Bernard (2004), la netnographie se rapproche du courant de la théorie enracinée. Fondée sur l'induction et sur un raisonnement analytique, elle oppose, compare, sélectionne les données. Elle se heurte, néanmoins, comme toute recherche en ligne aux limites suivantes :

- Elle s'appuie sur des déclarations, du narratif, du descriptif et non du comportement.

- Elle analyse des phénomènes en réseau et n'est donc pas exempte de biais liés à l'identité, la présentation de soi.

La popularité des blogueurs a une influence non négligeable sur leurs discours. La structure même des blogs où l'estimation de la longévité d'un billet repris de blog en blog est capitale ne conduirait-elle pas à une uniformisation de la pensée plutôt qu'au jaillissement de la singularité et de l'originalité ? Nous avons montré, dans notre recherche, que les zones d'incertitude ont été établies sur des discussions et des débats d'idées dans lesquels les blogueurs ont élaboré des analyses différentes. Ainsi, la netnographie permet de saisir des espaces de discussions libres et ouvertes.

La dernière limite à laquelle fait référence Bernard (2004) tient à la particularité des réseaux qui conduit à un changement de perspective. Si le moi numérique, l'identité que l'on veut présenter notamment dans un contexte professionnel peut influer sur le discours du blogueur, cette question est d'importance lorsque l'on procède à une contextualisation des données. Nous avons déterminé les contextes en nous appuyant sur l'élaboration d'une typologie des blogs de salariés. L'information présentée, sa nature peuvent varier selon qu'elle provient des blogs d'experts, des contestataires ou des opérationnels. Une seconde recherche pourrait amener une réponse à cette question. Les experts ou les opérationnels ont-ils une vision différente des métiers du Web et de leurs futurs possibles ? Les contestataires ont-ils une vision plus pessimiste que les connecteurs des avenirs possibles de ces métiers ? 


\section{Bibliographie}

Y. Bernard (2004), «La netnographie : une nouvelle méthode d'enquête qualitative basée sur les communautés virtuelles de consommation », Décision Marketing, 36, 49-62.

L. Boyer et A. Scouarnec (2008), «Des «possibles » environnementaux aux «possibles» organisationnels : quelle prospective des métiers de la publicité et de la communication », revue Management \& Avenir, 15, 104-119.

J. Füller J., G. Jawecki et H. Mühlbacher (2007), « Innovation creation by online basketball. Communities », Journal of Business Research, 60(1), p. 60-71

A. Grimand (2006), «Quand le Knowledge Management redécouvre l'acteur : la dynamique d'appropriation des connaissances en organisation », revue Management \& Avenir, 9, p. 141157.

R. Kozinets (2002), « The field behind the screen : using netnography for marketing research in online communities », Journal of Marketing Research, 39, (1), p 61 -72.

M. Mercanti-Guérin et A. d'Andria (2008), «Blogs de salariés : une remise en cause du management? », Les Etats généraux du management, « Management : tensions d'aujourd'hui et enjeux de demain », Palais du Luxembourg, Sénat, 17 octobre 2008.

R. Montil (2001), «Pour une approche renouvelée de la Prospective Métier», Revue Française de Gestion, 140.

W.H. Jr. Ross (2005), «What Every Human Resource Manager Should Know About Web Logs », SAM Advanced Management Journal, 4-13.

M. Tissioui (2008), «Essai de modélisation du processus de naissance des métiers : cas des métiers de soins », revue Management \& Avenir, 17.

E.C. Wenger, W.M. Snyder (2000), «Communauties of Practice : The Organizational Frontier », Harvard Business Review, 78 : 1, p. 139-145

\section{Webographie}

http://www.er.uqam.ca/nobel/gricis/actes/Bogues /Berard.pdf

http://caspeex.over-blog.com/article-1515200-6.html

http://www.christian-faure.net/2005/09/03/la-confiance-dans-management-des-connaissances/ http://loiclemeur.com/france/2004/10/_ellen_simonett.html

www.organisation20.com/

www.zazibao.com/ 